\title{
POWER SYSTEM CONTINGENCY RANKING BASED ON SHORT- TERM VOLTAGE STABILITY INDICES
}

\author{
J.D. PINZÓN ${ }^{\dagger}$ and D.G. COLOMÉ \\ Institute of Electrical Energy, National University of San Juan - CONICET, 5400 San Juan, Argentina \\ †jpinzon@iee.unsj.edu.ar
}

\begin{abstract}
C This paper presents a methodology to identify critical contingencies that produce shortterm voltage stability problems (STVS). The proposed methodology classifies the state of the power system for each contingency, assessing the voltage stability of the post-contingency dynamic response from the calculation of the maximal Lyapunov exponent (MLE) and dynamic voltage indices at each bus and the whole system. In order to determine the critical contingencies, the values of the indices and the results of the classification of the post-contingency state are statistically analysed. The methodology is tested in the New England 39-bus system, obtaining satisfactory results in relation to the identification not only of the most critical contingencies but also of vulnerable buses to voltage instability. New contributions of this work are the contingency classification methodology, the algorithm for calculating dynamic indices and the method of classification of the operating state as a function of the STVS problem magnitude.
\end{abstract}

Keywords-- Contingencies, Power systems, Lyapunov exponent, Short-term voltage stability.

\section{INTRODUCTION}

In recent years, power systems have been operated near their stability limits due to the progressive increase in demand, a lack of investment in transmission infrastructure and an increase in the complexity of the operation. In these conditions, an unexpected contingency, such as the loss of critical transmission lines or generators, can cause an avalanche of cascade outputs of components, the progressive increase of system stress, and a continuous decrease of bus voltages until exceeding the operation thresholds reaching voltage instability.

Voltage stability refers to the power system's ability to maintain voltage in acceptable values for the buses in the whole system under a disturbance from a given operating condition (Kundur et al., 2004).

Voltage stability has become a serious concern for the electricity sector, as several of the major collapses related to voltage instability (Glavic et al., 2012; Taylor, 1994), have caused large economic and social losses.

Voltage instability and collapse are usually caused by two types of disturbance: faults or contingencies and load variations, which are large and small disturbances. As regards the time in which it occurs, voltage instability can be a short-term (few seconds) or long-term (tens of seconds to minutes) phenomenon (Kundur et al., 2004). Large disturbances due to faults in electric grids or programmed disconnections impact the dynamic security of power systems. Therefore, the prior analysis of the severity of each contingency in offline studies allows transmission system operator (TSO) to be alerted to assess and implement preventive control strategies. Moreover, contingency analysis allows developing real-time corrective or emergency control strategies based on events to mitigate the effects of contingencies. The classification of dangerous contingencies or those which lead to instability according to the level of risk or se-verity is known as contingency ranking (Amjady, 2003).

Most known methods to determine the severity of contingencies are based on limits of active power flow. The DC power flow commonly used to classify contingencies according to the overload level of transmission lines (Brandwajn, 1988; Galiana, 1984), does not include the analysis of reactive power flow, which is one of the main variables in relation with voltage stability problems. In addition, contingency classification methods have been proposed based on the sensitivity analysis (Nam et al., 2000). However, these methods are subject to error due to the approximation that is made by not considering the higher order terms of Taylor series. The above methods assess contingencies with a static approach. In order to analyse the dynamic effects of the contingencies, timedomain methods have been proposed. In Tiwari and Ajjarapu $(2007,2016)$ the contingencies are analysed with a dynamic approach using indices to identify problems of fault-induced delayed voltage recovery (FIDVR), although these proposals do not analyse STVS nor fast voltage collapse.

Some methods have been proposed in Yang et al. (2018) and Zhu et al. (2017) to assess STVS through the calculation of indices, although these indices do not allow classifying in different levels the status of stability. For that reason, the efficient classification of contingencies that originates volt-age stability problems within the framework of the dynamic security assessment is still a subject for research.

Furthermore, some investigations such as Ni et al. (2017), Verdejo et al. (2015), Yibei et al. (2011) and Zheng et al. (2018) have demonstrated the chaotic nature of power systems, in which methods based on chaos theory have been used to identified stability problems. In Dasgupta et al. (2013) the fast voltage collapse is assessed through the estimation of the Lyapunov exponent computed by an algorithm that described problems with its parameters.

Studies of power system chaotic behaviour have demonstrated that it occurs not only for simplified power systems but also for more realistic ones with more than 
one hundred buses, in which in the analysed models were included the control devices and were modelled the dynamics of the power systems components and devices (Dasgupta et al., 2013; Ge et al., 2017; Verdejo et al., 2015).

In previous work of this research was analysed a Lyapunov-based method to assess fast voltage collapse from the time series using different embedding parameters, in which was identified chaotic attractors in a embedding space (Pinzón and Colomé, 2018).

In the analysis of the state-of-the-art is observed that there is no method to rank, in a hierarchical way, contingencies that produced STVS problems. From above, a new methodology of contingency ranking to be applied in offline studies based on the effects produced in voltage stability by contingencies is proposed in this paper. The methodology is characterized by classifying each contingency in different operating states through the computation of the maximal Lyapunov exponent (MLE) and dynamic voltage indices. The main contributions of this work are:

- The assessment of fast voltage collapses by means of the estimation of the MLE from time series of dynamic simulations.

- Dynamic performance assessment using four voltage recovery indices.

- A methodology to identify critical contingencies through a hierarchical computation of STVS indices.

The remainder of this paper is organized as follows. The problem of STVS and the mechanisms that produce instability are described in Section II. The methodology proposed for classifying contingencies is provided in Section III. The results are obtained applying the classification methodology in the New England 39-bus test system and these are shown in Section IV. Conclusions are presented at the end of the paper.

\section{PROBLEM STATEMENT}

\section{A. Short-term voltage stability (STVS)}

STVS occurs just few seconds after a disturbance and it is generally produced in operating conditions in which motor loads and electronically controlled loads constitute a large proportion of the local load. Thus, the dynamic load connected to the power system is increased by motors in the residential sector (pumps, air conditioners) and in the industrial sector cause increased vulnerability due to STVS (Potamianakis and Vournas, 2006). This type of instability is a matter of growing concern, but it is often ignored by the industry. Its analysis is still a challenge because of the difficulty in modelling components, fast interactions and dynamics, nonlinearity, high dimensionality and uncertainty in the operation of power systems. Therefore, it is a complex phenomenon in large power systems, and its assessment generates difficulties to the TSO (de Leon and Taylor, 2002; Pinzón and Colomé, 2017).

\section{B. Mechanisms of large-disturbance STVS}

Different mechanisms can cause voltage stability problems. In power systems with a high proportion of dynamic load when a fault occurs, it is usually followed by a voltage dip, which is characterized by the induction motors stall and if the curves of the mechanical and electric torque intersect after a short time, the voltage will recover to a value near the pre-disturbance voltage. The first mechanism is just the delay in the voltage recovery known as FIDRV, shown in Fig. 1, in which because of the dynamic response of different components under a fault and subsequent clearance in the New England 39bus test system on the line 16-17 unacceptable voltage values are presented. The FIDVR is a condition that increases the system stress, the improper operation of power equipment, mal-operation of protection relays and if the control systems do not operate adequately, it will produce outage of components (Glavic et al., 2012).

The second mechanism is the instability due to a fault in a transmission line and fault clearance, shown in Fig. 2 with a fault in the line $03-18$. This instability mechanism is related to the increase in total transmission impedance due to a topology change. Because of the increase in impedance, dynamic loads such as induction motors increase the reactive power consumed and the curves of the mechanical and electrical torque do not intersect after the disturbance, which causes the cascade motors stall at different buses and finally voltage instability.

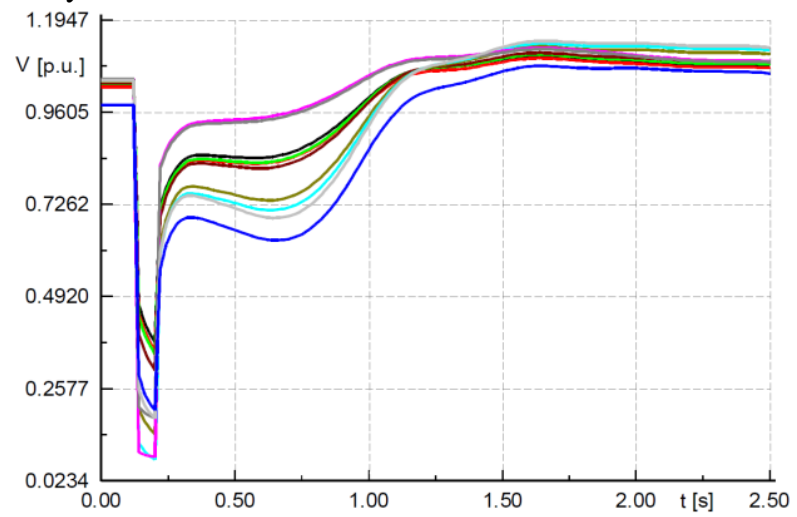

Fig. 1. Voltage profile (for buses 11-20 New England 39-bus system).

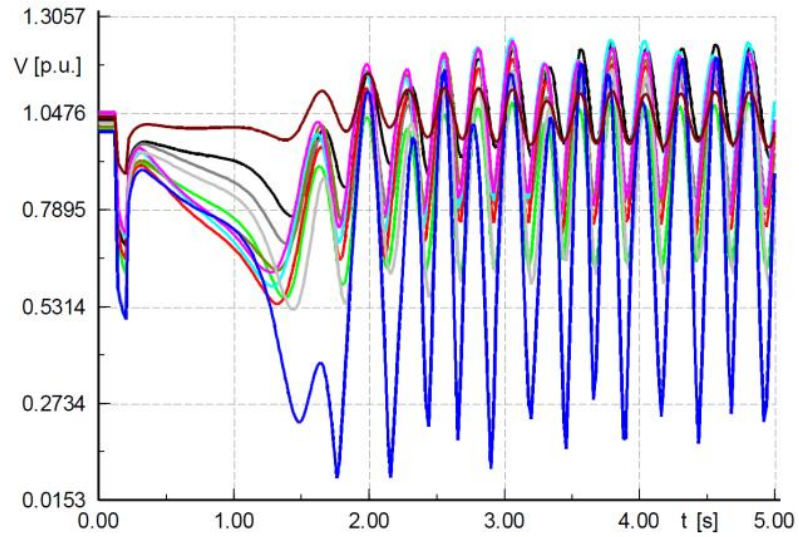

Fig. 2. Voltage profile (for buses 31-39 New England 39-bus system). 


\section{PROPOSED METHODOLOGY FOR CLASSIFYING CONTINGENCIES}

Voltage stability problems occur due to faults in the power system or to different system conditions and characteristics such as stress level, the load composition and the performance of excitation systems of generators and other control devices. In relation to voltage stability, the operating conditions and the evolution of the electrical variables after a contingency define the system state.

A methodology for classifying contingencies according to the level of voltage stability from the analysis of post-contingency dynamic response time series is proposed in Fig. 3. The methodology includes consideration of uncertainties in the operating state from a stochastic process that applies the Monte Carlo simulation (MC). Then, the classification of contingencies is performed from the limit values of dynamic indices (dynamic voltage index in each bus - $D V I_{b}$ and wide-area dynamic voltage index - WADVI) and the MLE.

The methodology for classifying and ranking contingencies includes the following steps:

a) Power system modelling, including fast dynamics components, such as Motors, AVR and generator dynamics.

b) Generation of cases $(\mathrm{Nc})$ with different load condition from a probability distribution function. Considering that the dynamic response of the power system is highly influenced by a large volume of uncertainties, it is a need to apply stochastic techniques such as MC to generate scenarios that cover a high variety of operating conditions.

c) Optimal power flow (OPF) calculation to obtain the dispatch of generators according to their load condition and the technical and economic constraints.

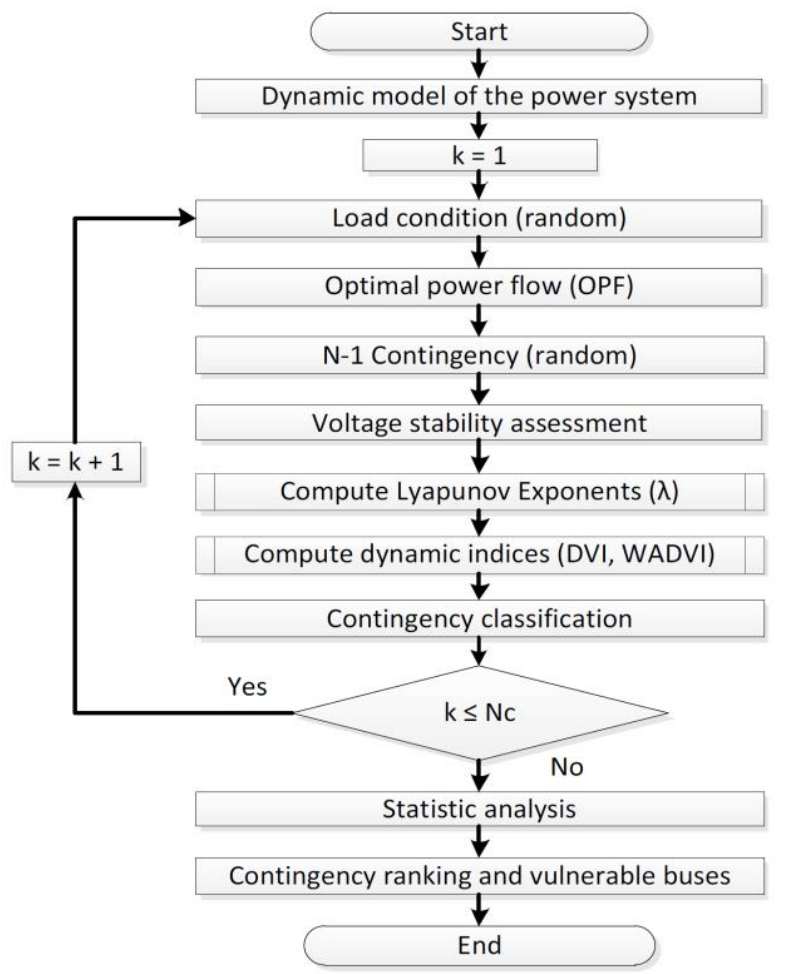

Fig. 3. Flowchart of the classification methodology. d) Random generation of N-1 contingencies in transmission lines (random fault location) or generators and timedomain dynamic simulation of contingencies.

e) MLE and dynamic voltage indices calculation during the simulation time $(5 \mathrm{~s})$.

f) Contingency classification based on the STVS postcontingency assessment through the analysis of the MLE and dynamic voltage indices.

g) Statistical processing of classification results of all simulated cases from the clustering of operating state for each contingency and the number of vulnerable cases per bus.

h) Identification of the most severe contingencies based on mean values of the WADVI index and the number of unstable cases produced by each contingency.

i) Identification of vulnerable buses based on the analysis of the mean of the $D V I_{b}$ index for each bus and according to the post-disturbance operation state.

\section{A. Lyapunov exponents}

Nonlinear analysis can be comparatively easy if the state space equations of the system dynamics are known but it is a difficult task when only the univariate measurement time series are known. The Lyapunov exponents (LE) are useful in a nonlinear analysis of time series. When exponents are positive by definition they are evidence of chaos and instability in the system (Rosenstein et al., 1993)

The LE quantifies the exponential divergence of trajectories, in the state space, with a small initial distance $(\epsilon)$ and it estimates the magnitude of chaos in a system. The presence of a positive characteristic exponent is evidence of instability. For exponents' calculation, all neighbor points of the time series that are very close to a particular reference point or initial point are searched. Then the increase in the distance during a relative time between the neighbor trajectory and the reference trajectory is calculated. The future distance is calculated using $|d(t)|=\epsilon e^{\lambda t}$ where $\lambda$ is the MLE. Specifically, the proposed algorithm in Hegger et al. (1999) and Rosenstein et al. (1993) provides a robust estimation of the MLE from a time series. With this algorithm the distance between trajectories is calculated using the coordinated time delay $(\tau)$. The distance between a reference trajectory $x_{i}$ and a neighbor trajectory $x_{j}$ after the relative time $t$ is defined by Eq. (1).

$$
\operatorname{dist}\left(x_{i}, x_{j} ; t\right)=\left|x_{i+t}-x_{j+t}\right| \text {. }
$$

Lyapunov exponents are computed using Eq. (2), which applies an algorithm for the search of the neighbor trajectories (Grassberger et al., 1991). In Eq. (2) $i$ is set, searching all neighbors $x_{j}$ of $x_{i}$ inside of a $\mathcal{U}_{i}$ neighborhood. Finally, the averages of distances between all neighbor trajectories and the reference trajectory $x_{i}$ as a function of $t$ are computed.

$$
S(t)=\frac{1}{T} \sum_{i=1}^{T} \ln \left(\frac{1}{\left|u_{i}\right|} \sum_{j \in u_{i}} \operatorname{dist}\left(x_{i}, x_{j} ; t\right)\right) .
$$



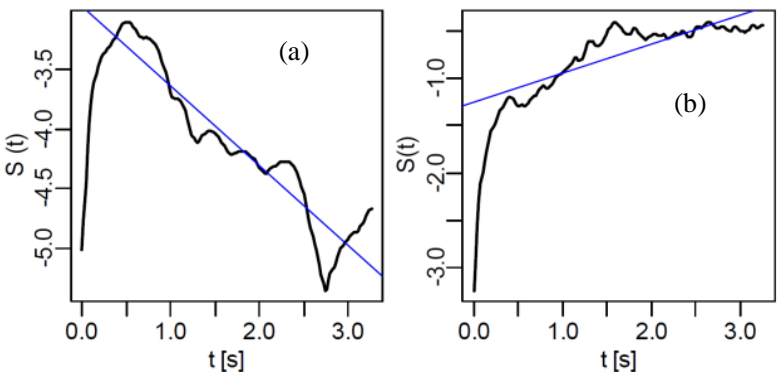

Fig. 4. Evolution of Lyapunov exponents and MLE a) Voltage recovery case $(\lambda=-0.67), b)$ Unstable case $(\lambda=0.306)$.

MLE is the slope of the line obtained from linear regression of the set of individual exponents. In this case, the time series $x(t)$ is equivalent to voltage time series $V(t)$. The spectrum of LEs for different time series is shown in Fig. 4. The voltage recovery case in (a) of Fig. 1 and unstable case in (b) of Fig. 2. The MLE obtained from the regression coefficients is negative for the case (a) and positive for the case (b). The positive value of MLE in case (b) indicates the chaos and the instability of the dynamic time series.

Concerning the number of samples in the analysis of the MLE in time series, one of the main advantages of the estimation algorithm used in this paper (Rosenstein et al., 1993 ) is that it requires a small set of samples for a robust estimation of the MLE. Besides, unlike the stability analysis performed for other dynamic systems, in power systems the exponential divergence of trajectories occurs in a few seconds, so using more simulation time would cause the MLE calculation to reflect the divergence in the swings after the collapse of the system (see Fig. 2) and not the divergence due to the transition from the power system stable equilibrium state to an unstable one.

\section{B. Algorithm for computing dynamic indices}

The dynamic analysis of bus voltage time series is made from computing voltage indices, which determine the deviation of the post-disturbance voltage values in relation to the pre-disturbance one during a specific time. The steps for computing these indices are following:

Step 1: Voltage index (VI) calculation for each bus and each time instant. The VI measures the relative deviation from the voltage with respect to the initial or predisturbance voltage. The VI is calculated applying the Eq. (3)

$$
V I_{b}^{t}=\frac{V_{b}^{0}-V_{b}^{t}}{V_{b}^{0}} ;\left\{\begin{array}{l}
b \in\left[1, N_{b}\right] \\
t \in\left[t_{c l}, t_{s}\right]
\end{array},\right.
$$

where $V_{b}^{0}$ is the pre-disturbance voltage, $N_{b}$ is the total number of buses, $t_{c l}$ is the fault clearing time and $t_{s}$ is the simulation time.

Step 2: Dynamic voltage index (DVI) calculation for each bus and each time window. The meaning of the DVI is the value below which the relative voltage is maintained for a specific number of cycles. The mobile time window $(\Delta t)$ is determined with the Eq. (4) to compute the DVI and the number of cycles according to the industrial criterion of Shoup et al. (2004), which is 20 cycles of nominal frequency. The DVI for each window is the minimum value of the VI values in that time period. The DVI is calculated using the Eq. (5), obtaining a DVI value for each window of the time series. The comparison of the evolution of $V(t)$, VI and DVI is shown in Fig. 5.

$$
\begin{gathered}
\tau=20 \frac{1}{f_{n o m}}=\Delta t_{m, n}=t_{n}-t_{m}, \\
D V I_{b}^{\Delta t_{m, n}}=\min V I_{b}^{t} ; \quad \begin{array}{c}
t \in\left[t_{m}, t_{n}\right] \\
\left\{\begin{array}{c}
t \\
t_{m}, t_{n} \in\left[\left(t_{c l}, t_{c l}+\tau\right),\left(t_{a}-\tau, t_{a}\right)\right]
\end{array}\right.
\end{array}
\end{gathered}
$$

Step 3: $D V I_{b}$ calculation for each bus applying the Eq. (6), which is the maximum DVI value of all windows that was computed in the step 2 .

$$
D V I_{b}=\max D V I_{b}^{\Delta t_{m, n}} .
$$

Step 4: Wide-area dynamic voltage index (WADVI) calculation or index of the whole system with the Eq. (7). The WADVI is defined as the maximal $D V I_{b}$ of the system.

$$
W A D V I=\max _{1 \leq b \leq N_{b}} D V I_{b} .
$$

\section{Contingency classification criteria}

The classification of different operation states according to stability levels allows determining the severity of contingencies. In this work, it is proposed to assess the stability due to a contingency using the MLE, which determines when the system is stable or unstable. If the result of the assessment is stable, the DVI and WADVI are calculated and then compared to a defined threshold to determine whether the state is: emergency, alert or normal. The value of WADVI is considered unaccepta-ble when it is higher than 0.2, according to the industrial criterion (Shoup et al., 2004), and the case in a state of emergency is classified. When the WADVI is between 0.1 and 0.2 is classified as a state of alert and when the WADVI is less than 0.1 is classified as a state of normal operation. This classification is shown in Fig. 6.

For example, the value of $\lambda$ is -0.679 and the WADVI is 0.295 for the case of the mechanism 1 of Fig. 1 . This case has a value of the MLE less than zero and a WADVI index higher than 0.2, therefore it is classified as an emergency case. In the case of a mechanism 2 of Fig. 2, the value of $\lambda$ is 0.306 , which is a positive value, then it is classified as an unstable case.

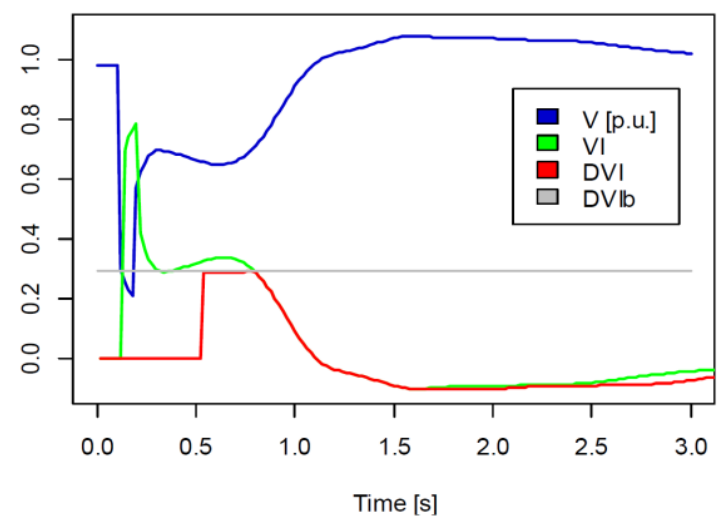

Fig. 5. Evolution of $V(t)$, VI and DVI. 


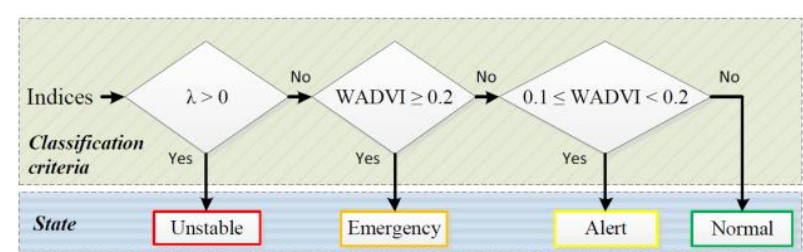

Fig. 6. Classification criteria.

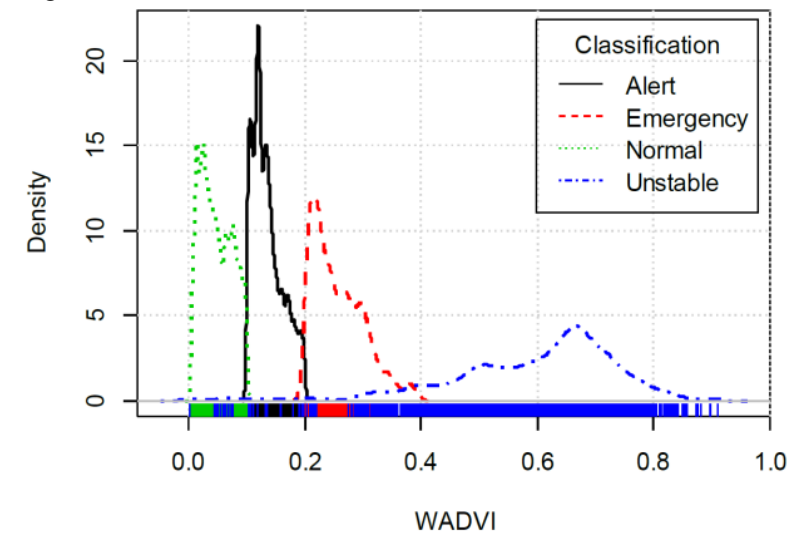

Fig. 7. Probability density according to the classification.

\section{SIMULATION RESULTS}

The developed methodology is tested in the New England 39-bus test system, which is a typical test system for stability studies. The time-domain dynamic simulations are performed in DigSilent Power Factory and the analysis of time series in $\mathrm{R}$ program

\section{A. Generation of scenarios}

A total of 10,000 different operating scenarios (Nc) were generated covering a wide variety of possible load/generation scenarios, considering different proportions between static loads and dynamic loads as small and large motors. A randomly selected contingency was applied for each operation scenario, in total, 45 different types of contingencies were simulated. They could be a fault in a transmission line or a generator outage. The fault clearing time of $0.08 \mathrm{~s}$, the simulation time of $5 \mathrm{~s}$ and the simulation step of $0.02 \mathrm{~s}$ was considered.

\section{B. Post-contingency state classification.}

The methodology proposed in this paper is applied to classify the 10,000 cases generated by MC. The results of the classification are shown in Table 1. The probability density of the WADVI is included in Fig. 7. Cases were grouped according to their post-contingency operation state. In fact, the shape biased towards higher values of the WADVI of the unstable state probability distribution. In addition, most cases in an unstable and emergency state have a WADVI higher than 0.19 and most of them higher than 0.3 .

Regarding the detection of false positives, it was reviewed the results of the dynamic simulations for different dynamic responses (swings with high and low damping, collapses at different times, voltage recovery problems, cases with fast recovery and other variations). The above analysis allows to validate that the MLE correctly identifies both the cases that reach the fast voltage collapse and the different dynamic changes.
Table 1. Classification of post-contingency operation state.

\begin{tabular}{cc}
\hline State & Number of cases \\
\hline Unstable & 1730 \\
Emergency & 589 \\
Alert & 2561 \\
Normal & 5120 \\
\hline
\end{tabular}

Table 2. Contingency ranking by WADVI mean.

\begin{tabular}{cccccc}
\hline \multicolumn{6}{c}{ Rank Contingency Mean } \\
\hline 1 & L16_24 & 0.60 & 9 & L16_19_a & 0.38 \\
2 & L16_21 & 0.58 & 10 & L14_15 & 0.30 \\
3 & L21_22 & 0.54 & 11 & L02_25 & 0.27 \\
4 & L23_24 & 0.50 & 12 & L01_02 & 0.27 \\
5 & L15_16 & 0.47 & 13 & L17_18 & 0.25 \\
6 & L16_17 & 0.45 & 14 & L26_29 & 0.24 \\
7 & L22_23 & 0.44 & 15 & L26_28 & 0.23 \\
8 & L16_19_b & 0.41 & 16 & L01_39 & 0.19 \\
\hline
\end{tabular}

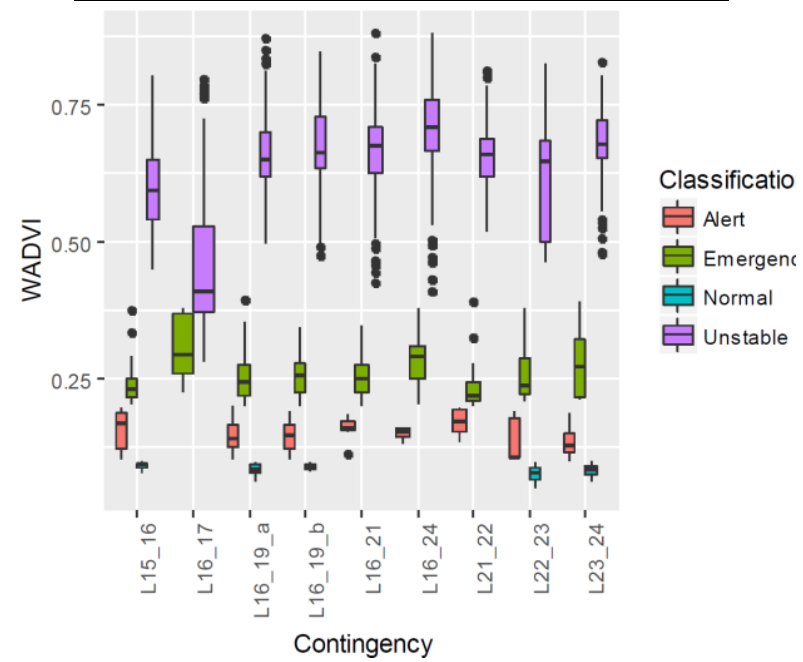

Fig. 8. WADVI boxplot for several contingencies.

\section{Contingency ranking}

The classification and ranking of contingencies are performed by statistical processing of the 10,000 scenarios according to the mean of the WADVI. In this way, contingencies with WADVI mean higher than or equal to 0.19 are presented in Table II. It is observed that the first four contingencies have mean values above 0.5 . The WADVI boxplot according to the state classification for contingencies with mean higher than 0.3 is shown in Fig. 8. The boxplot allows recognizing the statistical distribution of WADVI values for each contingency and state. Each contingency produces unstable, emergency, alert or normal cases. There is a high dispersion of values in the unstable cases for all selected contingencies; this is due to the fact of these contingencies produce more unstable than stable cases.

On the other hand, contingencies are ranked according to the percentage of cases in which they produce voltage instability. Thus, contingencies with more than $40 \%$ of unstable cases with respect to the analysed cases are shown in Table 3. In addition, the percentage of total cases in which each contingency produces an emergency and or alert state is presented.

Some contingencies such as faults in lines 16-17, 16$21,16-24$ and $21-22$ produce voltage instability in more than $70 \%$ of the cases, analysed in the 10,000 scenarios. 


\begin{tabular}{|c|c|c|c|c|c|c|}
\hline $\begin{array}{c}\text { Rank by } \\
\text { state } \\
\text { (by mean) }\end{array}$ & Contingency & $\begin{array}{l}\text { Total } \\
\text { cases }\end{array}$ & $\begin{array}{l}\text { Unsta- } \\
\text { ble (\%) }\end{array}$ & $\begin{array}{c}\text { Emer. } \\
(\%)\end{array}$ & $\begin{array}{c}\text { Alert } \\
(\%)\end{array}$ & $\begin{array}{c}\text { Nor- } \\
\operatorname{mal}(\%)\end{array}$ \\
\hline $1(6)$ & L16_17 & 193 & 97.4 & 2.6 & 0 & 0 \\
\hline $2(2)$ & L16_21 & 213 & 81.7 & 15.5 & 2.8 & 0 \\
\hline $3(1)$ & L16_24 & 198 & 77.3 & 20.2 & 2.5 & 0 \\
\hline $4(3)$ & L21_22 & 209 & 73.2 & 12.4 & 14.4 & 0 \\
\hline $5(5)$ & L15_16 & 193 & 68.9 & 11.4 & 16.6 & 3.1 \\
\hline $6(4)$ & L23_24 & 195 & 65.6 & 6.2 & 20.5 & 7.7 \\
\hline $7(7)$ & L22_23 & 176 & 63.6 & 7.4 & 4 & 25 \\
\hline $8(8)$ & L16_19_b & 196 & 49 & 12.8 & 34.2 & 4.1 \\
\hline $9(9)$ & L16_19_a & 215 & 43.3 & 19.1 & 32.6 & 5.1 \\
\hline
\end{tabular}

At the same time, in these contingencies, the sum of unstable cases plus those cases in a state of emergency plus cases in a state of alert are equivalent to a $100 \%$ of cases analysed for each contingency.

Comparing the ranking results according to the mean of the WADVI and according to the post-disturbance state, the contingencies of Table 3 correspond with the first contingencies with a mean higher than 0.3 of Table 2 , some of them in the same order. These contingencies are the most severe contingencies and produce $71 \%$ of the unstable cases in Table 1 . The ranking results based on the post-disturbance state allow verifying those the critical contingencies obtained from the ranking based on the WADVI mean. Some buses are more vulnerable to voltage instability, which is revealed with high values of $D V I_{b}$. The order for ranking buses is obtained based on the mean $D V I_{b}$ for the 10,000 scenarios. Buses with $D V I_{b}$ mean higher than 0.12 are shown in Table 4. The DVI of these buses is presented in boxplots in Fig. 9 classified according to their state of stability.

The system bus with the highest value of the $D V I_{b}$ index is identified for each case and this bus is associated with the operating state obtained from the classification. These results are used to rank in Table 5 those buses with the highest $D V I_{b}$ according to the number of cases that produces voltage instability, which is the result of the classification methodology. It is observed that all vulnerable buses are load buses and added cases of instability accumulate more than $75 \%$ of the cases classified as unstable in Table 1. Seven nodes coincide between the buses selected in Table 5 and the buses selected in Table 4 , which represent the buses with the highest vulnerability to voltage instability. The results of the identification and ranking of vulnerable buses by the post-contingency state allow verifying the vulnerable buses identified from the ranking by the $D V I_{b}$ mean.

Table 4. Ranking of vulnerable buses by $D V I_{b}$ mean

\begin{tabular}{ccc}
\hline Rank & Bus & Mean \\
\hline 1 & 24 & 0.136 \\
2 & 15 & 0.135 \\
3 & 16 & 0.135 \\
4 & 17 & 0.129 \\
5 & 27 & 0.127 \\
6 & 21 & 0.125 \\
7 & 18 & 0.124 \\
8 & 26 & 0.123 \\
9 & 28 & 0.122 \\
\hline
\end{tabular}

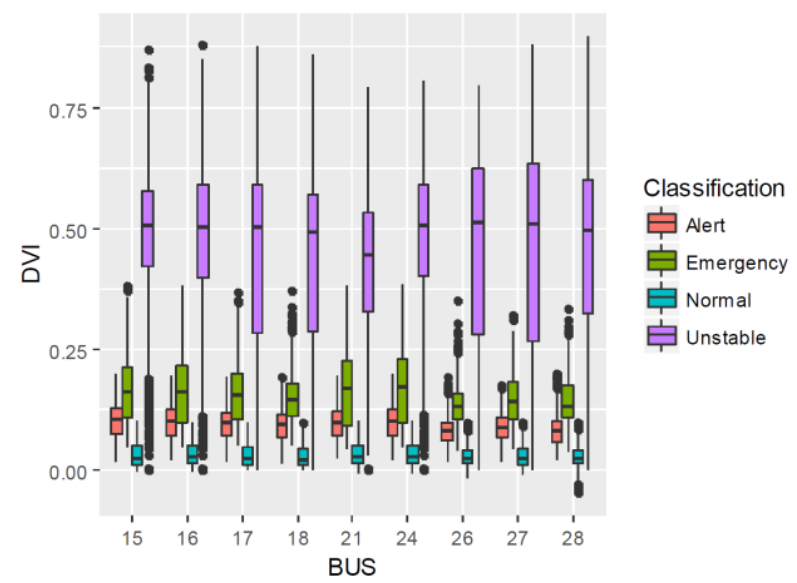

Fig. 9. Boxplot DVI for vulnerable buses.

Table 5. Ranking of vulnerable buses by state

\begin{tabular}{ccccc}
\hline $\begin{array}{c}\text { Rank by state (by } \\
D V I_{b} \text { mean) }\end{array}$ & Bus & Unstable & Emergency & Alert \\
\hline $1(9)$ & 28 & 255 & 49 & 97 \\
$2(8)$ & 26 & 197 & 0 & 0 \\
$3(2)$ & 15 & 192 & 82 & 405 \\
$4(5)$ & 27 & 187 & 0 & 70 \\
$5(1)$ & 24 & 139 & 83 & 224 \\
$6(6)$ & 21 & 114 & 11 & 6 \\
$7(29)$ & 8 & 80 & 4 & 26 \\
$8(11)$ & 29 & 72 & 18 & 49 \\
$9(3)$ & 16 & 66 & 3 & 3 \\
\hline
\end{tabular}

\section{Time considerations}

The simulation results obtained in the previous sections were developed in a computer with a processor AMD Athlon $^{\mathrm{TM}}$ II X4 - $3 \mathrm{GHz}$ and 4 GB RAM. The time required to compute the MLE in all buses of the test system for each scenario is $0.11 \mathrm{~s}$. The time to compute the dynamic indices for each scenario is $0.09 \mathrm{~s}$. These times allow identifying the stability state and after computing in a large amount of cases determine the critical contingencies.

\section{CONCLUSIONS}

In this paper, a methodology for classifying contingencies that produce short-term voltage stability problems is presented. As part of the methodology, a voltage stability classification method is introduced, which allows to classify the post-contingency operation state in one of the following states: normal, alert, emergency or unstable. The classification is based on the MLE and dynamic voltage indices. These indices allow assessing different voltage problems such as fast voltage collapse and delayed voltage recovery.

The methodology based on dynamic indices allows determining critical contingencies. These results were verified with those results obtained from the analysis of contingencies according to the classification of the postdisturbance state. Furthermore, the combined analysis of $D V I_{b}$ mean values for each bus and those buses from which the WADVI is classified according to the postcontingency operation state allows identifying vulnerable buses to voltage instability.

This methodology has been successfully applied for the analysis of contingencies in a test system. 
The most severe contingencies that produce voltage stability problems and the vulnerable buses to instability were identified applying the proposed methodology. In addition, the prior ranking of contingencies will provide useful information for dynamic security studies and will allow defining alerts to operators and strategies of preventive and corrective control based on events. At the same time, the identification of vulnerable buses will allow to determine critical areas and effective location of control devices for voltage support.

\section{REFERENCES}

Amjady, N. (2003) "Dynamic voltage security assessment by a neural network based method," Electric Power Systems Research, 66, 215-226.

Brandwajn, V. (1988) "Efficient bounding method for linear contingency analysis," IEEE Transactions on Power Systems, 3, 38-43.

Dasgupta, S., Paramasivam M., Vaidya U. and Ajjarapu V. (2013) "Real-Time Monitoring of Short-Term Voltage Stability Using PMU Data," IEEE Transactions on Power Systems, 28, 3702-3711.

de Leon, J.A.D. and Taylor C.W. (2002) "Understanding and solving short-term voltage stability problems," IEEE Power Engineering Society Summer Meeting, Chicago USA.

Galiana, F.D. (1984) "Bound Estimates of the Severity of Line Outages in Power System Contingency Analysis and Ranking," IEEE Transactions on Power Apparatus and Systems, PAS-103, 2612-2624.

Ge, H., Guo Q., Sun H., Wang B., Zhang B., Liu J., Yang Y.and Qian F. (2017) "An Improved Real-time Short-term Voltage Stability Monitoring Method Based on Phase Rectification," IEEE Transactions on Power Systems, 33, 1068-1070.

Glavic, M., Novosel D., Heredia E., Kosterev D., Salazar A., Habibi-Ashrafi F.and Donnelly M. (2012) "See It Fast to Keep Calm: Real-Time Voltage Control Under Stressed Conditions," IEEE Power and Energy Magazine, 10, 43-55.

Grassberger, P., Schreiber T. and Schaffrath C. (1991) "Nonlinear time sequence analysis," International Journal of Bifurcation and Chaos, 1, 521-547

Hegger, R., Kantz H. and Schreiber T. (1999) "Practical implementation of nonlinear time series methods: The TISEAN package," Chaos: An Interdisciplinary Journal of Nonlinear Science, 9, 413-435.

Kundur, P., Paserba J., Ajjarapu V., Andersson G., Bose A., Canizares C., Hatziargyriou N., Hill D., Stankovic A., Taylor C., Van Cutsem T.and Vittal V. (2004) "Definition and Classification of Power System Stability IEEE/CIGRE Joint Task Force on Stability Terms and Definitions," IEEE Transactions on Power Systems, 19, 1387-1401.

Nam, H.-K., Kim Y.-K., Shim K.-S. and Lee K.Y. (2000) "A new eigen-sensitivity theory of augmented matrix and its applications to power system stability analysis," IEEE Transactions on Power Systems, 15, 363-369
Ni, J., Liu L., Liu C., Hu X. and Li S. (2017) "Fast fixedtime nonsingular terminal sliding mode control and its application to chaos suppression in power system," IEEE Transactions on Circuits and Systems II: Express Briefs, 64, 151-155.

Pinzón, J.D. and Colomé D.G. (2017) "Voltage stability assessment using synchrophasor measurements: Trends and development," IEEE PES Innovative Smart Grid Technologies Conference - Latin America (ISGT Latin America)

Pinzón, J.D. and Colomé D.G. (2018) “Chaos in Power Systems: Towards Short-term Voltage Stability Analysis," IEEE PES Transmission \& Distribution Conference and Exhibition- Latin America (T\&D$L A)$

Potamianakis, E.G. and Vournas C.D. (2006) "Shortterm voltage instability: effects on synchronous and induction machines," IEEE Transactions on Power Systems, 21, 791-798.

Rosenstein, M.T., Collins J.J. and De Luca C.J. (1993) "A practical method for calculating largest Lyapunov exponents from small data sets," Physica D: Nonlinear Phenomena, 65, 117-134.

Shoup, D.J., Paserba J.J. and Taylor C.W. (2004) “A survey of current practices for transient voltage dip/sag criteria related to power system stability," IEEE PES Power Systems Conference and Exposition.

Taylor, C.W. (1994) Power system voltage stability, McGraw-Hill.

Tiwari, A. and Ajjarapu V. (2007) “Contingency assessment for voltage dip and short term voltage stability analysis," IREP Symposium - Bulk Power System Dynamics and Control - VII Revitalizing Operational Reliability.

Tiwari, A. and Ajjarapu V. (2016) "Addressing short term voltage stability problem - Part I: Challenges and plausible solution directions," IEEE/PES Transmission and Distribution Conference and Exposition $(T \& D)$

Verdejo, H., Kliemann W. and Vargas L. (2015) “Application of linear stability via Lyapunov exponents in high dimensional electrical power systems," International Journal of Electrical Power \& Energy Systems, 64, 1141-1146.

Yang, H., Zhang W., Chen J. and Wang L. (2018) "PMUbased voltage stability prediction using least square support vector machine with online learning," Electric Power Systems Research, 160, 234-242.

Yibei, W., Man L., Yanting X. and Hougui C. (2011) "Research on chaos phenomena in power system," Power Engineering and Automation Conference (PEAM), 2, 453-456.

Zheng, L., Liu Z., Shen J. and Wu C. (2018) "Very shortterm maximum Lyapunov exponent forecasting tool for distributed photovoltaic output," Applied Energy, 229, 1128-1139.

Zhu, L., Lu C., Liu Y., Wu W. and Hong C. (2017) "Wordbookbased light-duty time series learning 
machine for short-term voltage stability assessment," IET Generation, Transmission \& Distribution, 11, 4492-4499.
Received July 10, 2018.

Sent to Guest Editor October 26, 2018.

Accepted August 12, 2019.

Recommended by Guest Editor Ma. Belén D'Amico 